CLINICAL STUDY

\title{
Cognitive outcome of offspring from dexamethasone-treated pregnancies at risk for congenital adrenal hyperplasia due to 21-hydroxylase deficiency
}

\author{
Heino F L Meyer-Bahlburg, Curtis Dolezal, Rita Haggerty, Michael Silverman ${ }^{1}$ and Maria I New ${ }^{2}$ \\ NYS Psychiatric Institute and Department of Psychiatry, Columbia University, 1051 Riverside Drive, NYSPI Unit 15, New York, New York 10032, USA, \\ Departments of ${ }^{1}$ Psychiatry and ${ }^{2}$ Pediatrics, Mount Sinai School of Medicine, New York, New York 10029, USA \\ (Correspondence should be addressed to H F L Meyer-Bahlburg; Email: meyerb@nyspi.columbia.edu)
}

\begin{abstract}
Objectives: To test whether dexamethasone (DEX) treatment in pregnancies at risk for congenital adrenal hyperplasia $(\mathrm{CAH})$ impairs cognitive functioning in the offspring.

Design: Observational follow-up of prenatally DEX-exposed offspring and controls.

Methods: Study 1 included 140 children aged 5-12 years: 67 DEX-exposed (long-term: eight CAH girls) and 73 unexposed (with 15 CAH girls). Study 2 included 20 participants aged 11-24 years: seven DEX-exposed (long-term: one $\mathrm{CAH}$ woman) and 13 unexposed (with four CAH women). Neuropsychological testing was done in hospital settings or at patients' homes. Data analysis aimed at maximizing detection of the effects of DEX exposure.

Results: The vast majority of group comparisons were not marginally or conventionally significant. The few significant findings on short-term prenatal DEX exposure suggested more positive than adverse outcomes. By contrast, few significant findings in females with CAH and long-term DEX exposure indicated slower mental processing than in controls on several neuropsychological variables, although partial correlations of DEX exposure duration with cognitive outcome did not corroborate this association.

Conclusions: Although our studies do not replicate a previously reported adverse effect of short-term prenatal DEX exposure on working memory, our findings on cognitive function in CAH girls with long-term DEX exposure contribute to concerns about potentially adverse cognitive after effects of such exposure. Yet, our studies are not definitive, and replications in larger samples are required.
\end{abstract}

European Journal of Endocrinology 167 103-110

\section{Introduction}

46,XX newborns affected by classical congenital adrenal hyperplasia (CAH) due to 21-hydroxylase deficiency (21-OHD) show variable masculinization of the genitalia. They are, therefore, at increased risk of erroneous assignment to the male gender or, if assigned to the female gender, of feminizing genital surgery with its attendant risks to later sexual functioning $(1,2)$. To prevent these sequelae of the $\mathrm{CAH}$ condition, dexamethasone (DEX) treatment in CAH-risk pregnancies was introduced in 1978 by Forest in France and in 1986 by New in the USA $(3,4)$. The treatment has indeed been shown to be highly efficacious in reducing genital masculinization in 46,XX fetuses, if begun by the 8th week postconception and used consistently throughout pregnancy $(5,6)$. Yet, this approach to prevention has become increasingly controversial because animal studies on the effects of fetal exposure to high-dose DEX suggest potentially adverse side effects on brain development and, especially, memory function, along with 'programming' of the hypothalamic-pituitary-adrenal axis (HPAA) involving potentially long-term adverse effects on blood pressure, glucose regulation, and other functions $(5,7,8)$; shortterm HPAA effects have also been shown in humans (9). An additional concern is the fact that for DEX treatment to be effective it has to begin before the sex and $\mathrm{CAH}$ diagnosis of the fetus can be established with current ascertainment methods. This implies that the treatment is not limited to 46,XX fetuses with definitive diagnosis of classical 21-OHD CAH. It also exposes to DEX CAH-affected 46,XY fetuses and CAH-unaffected fetuses of both sexes, until fetal sex and CAH status can be determined either via amniocentesis (as of gestational weeks 15-18) or via chorionic villus sampling (as of gestational weeks 10-12). Thus, in such cases, the relatively short-term exposure to DEX may still carry risks without providing any benefits. The use of cell-free fetal Y-chromosomal DNA in maternal plasma as a new noninvasive technique for fetal sex determination early in pregnancy may now allow the exclusion of 
males from this treatment altogether (5), but it was not available for the samples described here.

Recent reviews have summarized a few and as yet unreplicated pertinent findings concerning potential adverse psychological side effects in prenatally DEXexposed offspring from a few small-scale psychological follow-up studies available on humans (5, 6). An early pilot study from our team showed increased shyness and inhibition in DEX-exposed children (10), but a larger follow-up study using several parent-administered, standardized developmental questionnaires on 174 prenatally DEX-exposed children (including 48 with $\mathrm{CAH}$ ) and 313 unexposed children (including 195 with CAH) did not show any effects of short-term or long-term prenatal DEX exposure on standard scales of motor, cognitive, and social development (11). More recently, a Swedish study by Hirvikoski et al. compared 26 DEXexposed children aged 7-17 years (four girls with CAH, five boys with $\mathrm{CAH}$, seven non-CAH boys, and ten nonCAH girls) with 35 non-CAH controls (15 boys and 20 girls) matched for age and sex, by direct examination of children by neuropsychological tests. The authors found no effects of prenatal DEX exposure on intelligence, handedness, memory encoding, or long-term memory, but among the short-term-treated, CAH-unaffected children had significantly poorer performance than controls on a test of verbal working memory as well as lower questionnaire scores on self-perceived scholastic competence and increased social anxiety (12). The parents described these children as more sociable than controls, and there were no significant differences in psychopathology, behavioral problems, adaptive functioning, parents' reports of 'any academic or other problems in school', or placement in a special class/special school(13).

The current report focuses exclusively on cognitive functioning in offspring from DEX-exposed pregnancies and controls. If early-onset prenatal DEX exposure has indeed adverse effects on the developing brain in humans as deduced from the animal data, one could expect impairments of specific cognitive functions, such as working memory, as well as of even broader indicators, such as intelligence or educational attainment. Here, we will report findings from two neuropsychological follow-up studies on DEX-exposed offspring and controls: Study 1 on 5- to 12-year-old children and Study 2 on a small feasibility sample of adolescents and adults.

\section{Subjects and methods}

\section{Study population}

Study 1 was conducted as one of several USA-wide follow-up studies on offspring from DEX-treated CAH-risk pregnancies. The main source of participating children was the prenatal diagnosis database of one of the authors (M I New), which included prenatally DEX-exposed and DEX-unexposed boys and girls with and without $\mathrm{CAH}$ (4), all from families with at least one CAH-affected index case (sibling, parent, or close relative). The DEX treatment itself had been under the care of the mother's local obstetrician/gynecologist. Additional unexposed $\mathrm{CAH}$ children came from the same author's database for other medical CAH studies. Following our preceding questionnaire study of the cognitive and motor development of prenatally DEX-exposed children from infancy to 12 years of age in a USA-wide sample (11), we conducted a comprehensive face-to-face investigation of behavioral aftereffects of prenatal DEX exposure in 140 children aged 5-12 years (for gender identity-related findings from that study, see (14)). Because of funding limits, only a part sample of the eligible children evaluated in the preceding study could be tested in the current project. Apart from requiring a minimum age of 5 years, the major selection criterion was parental consent (during the data collection period from June 1998 to November 2000) either to the family's evaluation visit in the senior author's (M I New) hospital setting in New York City or to the study team's evaluation visit in the family's hometown. For the latter option, we favored geographical areas with several families that were able to participate, in order to minimize the travel expenses of our team. Among the children without $\mathrm{CAH}$, we selected more females than males in order to increase the statistical power of comparisons for subtle DEX effects, if any, on (androgen-influenced) gender behavior in females (a topic not covered in the current paper). We also aimed at achieving a balanced distribution between DEX-exposed and DEX-unexposed children.

The final sample included 140 children aged 5-12 years: 67 with DEX exposure (long-term: eight $\mathrm{CAH}$ girls (seven with the salt-wasting (SW) variant and one with the simple-virilizing (SV) variant); short-term: eight CAH boys (seven SW and one SV), 35 non-CAH girls, and 16 non-CAH boys) and 73 unexposed (15 CAH girls (13 SW and two SV), 11 CAH boys (ten SW and one SV), 31 non-CAH girls, and 16 non-CAH boys). In terms of ethnicity, 130 children were white, six Hispanic, two African-American, one Asian, and one other. We were able to obtain the start and end date of DEX treatment for 65 of the 67 DEX-exposed children (it was unavailable for one boy with $\mathrm{CAH}$ and one nonCAH girl). Although in principle 46,XX fetuses with CAH should be DEX treated throughout pregnancy and the others only for short periods in early pregnancy, the calculated duration of actual DEX exposure was quite variable, depending on maternal access to and compliance with DEX treatment during pregnancy: the two lowest duration scores in girls with $\mathrm{CAH}$ were 9 and 19 weeks, and the seven longest duration scores among the non-CAH children ranged from 23 to 39 weeks. Nevertheless, as the mean and median of DEX duration for the DEX-exposed girls with $\mathrm{CAH}$ are significantly and very markedly higher than those of the other DEX-exposed subgroups, we will use the denotation 
'long-term' for the DEX exposure of girls with CAH and 'short-term' for the DEX exposure of the other subgroups throughout this article. Mean midparental education (15) was 5.50 (between at least 1 year of college or specialized training, and standard college or university graduation, but not a graduate degree); the lowest score was 3.5 (just below high school graduate), which applied to nine children. Thus, in comparison with national norms, the sample was somewhat elevated in socioeconomic level. For subjects and methods of Study 2, see Supplementary Study (see section on supplementary data given at the end of this article).

\section{Procedures}

The face-to-face evaluations took place either in an office of the senior author's (M I New) department or in a suitable room of the family's home or a nearby hotel. Written informed consent was obtained from all participating mothers and assent from the older children, after the appropriate institutional review boards had approved the study. Participants received \$100 per family for their study participation, and families traveling to New York city were reimbursed for their travel expenses.

\section{Assessments}

Neuropsychological tests were done as part of a comprehensive assessment protocol involving semistructured interviews and systematic play observations with the child and a demographic interview with a parent. The tests were done early in the sequence of procedures in order to minimize the child's fatigue. Cognitive development was assessed with the Kaufman Assessment Battery for Children (K-ABC) $(16,17)$ and the Wide Range Assessment of Memory and Learning (WRAML) (18). The K-ABC is an intelligence test that includes three global scales of problem solving and information processing, Sequential Processing (including short-term memory tasks that require immediate recall of visual or auditory stimuli), Simultaneous Processing (with subtests composed of visual spatial stimuli), and Mental Processing Composite (a combination of the other two), and one global scale of acquired factual knowledge, Achievement (including measures of reading skills and other subtests that require verbal responses to either pictures or questions). The WRAML provides four 'learning indexes': Verbal Memory (use of language in memory tasks that vary in semantic complexity), Visual Memory (visual memory tasks that vary in meaningfulness), Learning (performance over repeated trials), and General Memory (composite of the first three scales). Both the K-ABC global scales and the WRAML indexes are expressed as standardized age-based norm scores with a population mean of 100 and a s.D. of 15 . The subtests of the K-ABC Mental Processing Scales provide a scaled score with a mean of 10 and a s.D. of 3; the K-ABC Achievement subtests yield scaled scores with a mean of 100 and a S.D. of 15. The testing was performed by research assistants with a bachelor degree in psychology, who were trained in the administration of the study tests by a collaborating neuropsychologist (R Haggerty), and their performance was monitored by the study coordinator, a doctorate-level senior clinical psychologist. These research assistants did not participate in the recruitment procedures and did not know the CAH and DEX exposure status of the children they tested.

\section{Data analysis}

To maximize our chances of detecting adverse effects of DEX treatment, we used four approaches: i) DEXexposed and DEX-unexposed participants were compared based on neuropsychological test scores by independent samples $t$-tests. $t$-tests and Cohen's $d$ (using pooled variances) were performed separately for girls with $\mathrm{CAH}$, girls without $\mathrm{CAH}$, boys with $\mathrm{CAH}$, and boys without $\mathrm{CAH}$, as well as for non-CAH girls and boys combined. (As two of the K-ABC subtests, Photo Series and Reading/Understanding, are not applicable to our youngest age groups, the respective subsample sizes are reduced, as listed in Supplementary Table 1, see section on supplementary data given at the end of this article.) Age and midparental education were considered as covariates in these analyses but not included because no significant differences were found between any comparison groups, and the test scores were age-based norm scores. ii) Hierarchical regression analyses were performed separately for boys and girls. Both DEX (yes/no) and CAH (yes/no) were included in the initial regression model to assess the main effect of DEX treatment, controlling for CAH status. The DEX by $\mathrm{CAH}(\mathrm{DEX} \times \mathrm{CAH})$ interaction was then added to the regression model to test whether neuropsychological performance was associated with DEX treatment differently between $\mathrm{CAH}$ and non-CAH participants. iii) Because of the variability of DEX exposure duration, we performed partial correlations to test for an association between neuropsychological scores and the duration of DEX treatment (in weeks). Initial correlations included all DEX-treated participants and adjusted for CAH status (non-affected, 0; affected, 1) and gender (boy, 1; girl, 2). Additional correlations were performed separately for $\mathrm{CAH}$ and non-CAH participants, adjusting for gender. iv) Finally, as in the Swedish study (12), all three sets of analyses, except those with Achievement and its subtests as outcomes, were redone as regression analyses adjusting for intelligence quotient (IQ; operationalized as $\mathrm{K}-\mathrm{ABC}$ Achievement) to test whether controlling for IQ noticeably affected the results.

Statistical analyses were conducted using SPSS for Windows release 18.0.0. In line with our detection focus, we report both marginal $(P \leq 0.10)$ and conventional $(P \leq 0.05)$ significance levels without adjustment for multiple comparisons. 


\section{Results}

In regard to Study 1, Table 1 presents the mean and s.D. of age, midparental education, and the major neuropsychological outcome variables as well as their effect sizes for the DEX-exposed vs the DEX-unexposed separately for girls with $\mathrm{CAH}$, girls without $\mathrm{CAH}$, boys with $\mathrm{CAH}$, and boys without CAH. Only three of the $40 t$-tests on neuropsychological test variables reached marginal significance $(0.05<P \leq 0.10)$ and none reached conventional significance $(P \leq 0.05)$. Girls with $\mathrm{CAH}$ and long DEX exposure performed (marginally) more poorly on the K-ABC Mental Processing Composite. Non-CAH boys with short-term DEX exposure performed (marginally) better on $\mathrm{K}-\mathrm{ABC}$ Sequential Processing, and the same held true for non-CAH boys and girls combined.

Of the 16 hierarchical regression analyses, nine reached one or more results of (marginal or conventional) statistical significance. For girls, analyses of the K-ABC global scores yielded no main effects for DEX treatment but marginal effects for $\mathrm{CAH}$ (girls with $\mathrm{CAH}$ scoring lower than non-CAH) and conventionally significant $(P \leq 0.05) \mathrm{CAH} \times \mathrm{DEX}$ interactions on two K-ABC variables, Sequential Processing and the Mental Processing Composite (which includes the former variable). These interactions indicate that among the CAH-affected girls, the (long-term) DEX-exposed ones scored lower (worse) than the unexposed, while among the non-CAH girls, the (short-term) DEX-exposed ones scored higher (better) than the unexposed. Analyses of the WRAML indexes yielded neither any significant main effects nor any significant interaction effects.

For boys, analyses of the K-ABC global scores showed no main effects for $\mathrm{CAH}$ and no significant $\mathrm{CAH} \times \mathrm{DEX}$ interactions. However, the main effects for DEX were marginally significant $(P \leq 0.10)$ for the three variables: Sequential Processing, Mental Processing Composite (which includes the former), and Achievement; the mean scores for prenatally (short-term) DEX-exposed boys were higher (better) than those for the unexposed. None of the analyses of the WRAML indexes for boys reached marginal or conventional significance.

Of the 24 partial correlations for global test variables within DEX-exposed children (not shown), only one reached conventional significance. Among non-CAH children, DEX treatment duration correlated positively $\left(r_{\mathrm{p}}=0.33 ; \mathrm{df}=47 ; \mathrm{P}=0.020\right)$ with WRAML Visual Memory. Two correlations were marginal: DEX treatment duration correlated positively with WRAML Visual Memory $\left(r_{\mathrm{p}}=0.24 ; \mathrm{df}=61 ; P=0.063\right)$ for the total sample and WRAML General Memory Index $\left(r_{\mathrm{p}}=0.27 ; \mathrm{df}=47 ; P=0.059\right)$ for the non-CAH children.

Supplementary Table 1 (see section on supplementary data given at the end of this article) presents the respective data for the 13 individual K-ABC subtests that apply to the age group examined in this study, arranged by their respective global score categories:
Sequential Processing, Simultaneous Processing, and Achievement. Only nine of the $65 t$-tests on K-ABC subtests reached marginal or conventional significance. Girls with CAH and (long-term) DEX exposure performed (marginally) less well on Hand Movements (a subtest for Sequential Processing) and Spatial Memory (Simultaneous Processing). Non-CAH girls with (shortterm) DEX exposure performed significantly less well on Faces \& Places (a subtest of Achievement). Boys with CAH did not show an effect of (short-term) DEX exposure. Non-CAH boys with (short-term) DEX exposure performed significantly better than DEX-unexposed boys on Number Recall and Word Order (both subtests of Sequential Processing) and marginally better on Reading/Decoding and Reading/Understanding. Non-CAH boys and girls combined with (short-term) DEX exposure performed significantly better than the unexposed on Number Recall and marginally better on Word Order.

Of the 13 hierarchical regression analyses for girls, six variables showed results of conventional or marginal significance; two of these were Sequential Processing subtests, three Simultaneous Processing subtests, and one an Achievement subtest. $\mathrm{CAH}$ girls as a group performed less well than non-CAH girls on Triangles (conventional significance), Word Order (marginal significance), and Photo Series (conventional significance). $\mathrm{CAH} \times \mathrm{DEX}$ interactions reached marginal significance on three variables and conventional significance on one. On Hand Movements and Spatial Memory, DEX-exposed CAH girls performed less well than DEX-unexposed CAH girls, while DEX-exposed non-CAH girls performed slightly better than DEX-unexposed non-CAH girls. The reverse pattern emerged on Faces \& Places. No main effects for DEX exposure became significant.

Of the 13 hierarchical regression analyses for boys, five variables showed results of conventional or marginal significance; two of these were Sequential Processing subtests, one a Simultaneous Processing subtest, and two Achievement subtests. On Number Recall, CAH boys performed less well than non-CAH boys, and all five variables (Number Recall, Word Order, Photo Series, Reading/Decoding, and Reading/ Understanding) showed better performance in boys with DEX exposure. None of the CAH $\times$ DEX interactions reached significance.

Of the 39 partial correlations for the K-ABC subtests (not shown in Supplementary Table 1, see section on supplementary data given at the end of this article), three were conventionally significant. DEX treatment duration correlated positively with Faces \& Places for the total sample $\left(r_{\mathrm{p}}=0.29 ; \mathrm{df}=61 ; P=0.022\right)$ as well as for non-CAH children $\left(r_{\mathrm{p}}=0.30 ; \mathrm{df}=47 ; P=0.037\right)$ and negatively with Reading/Understanding for $\mathrm{CAH}$ children $\left(r_{\mathrm{p}}=0.87 ; \mathrm{df}=5 ; P=0.011\right)$, but the small sample size of the latter raises doubts in its utility. 
Table 1 Study 1: comparison of DEX-unexposed (non-DEX) and DEX-exposed (DEX) children on age, midparental education, and neuropsychological tests by $t$-test and multiple regression. $d$ effect size (DEX-non-DEX); negative $d$ values indicate poorer performance of DEX-exposed children.

\begin{tabular}{|c|c|c|c|c|}
\hline & Non-DEX mean (S.D.) & DEX mean (S.D.) & $\boldsymbol{d}$ & $\boldsymbol{P}$ \\
\hline \multicolumn{5}{|l|}{ Girls with $\mathrm{CAH}$} \\
\hline$n$ & 15 & 8 & & \\
\hline Midparental education & $5.20(0.94)$ & $5.12(1.09)$ & -0.08 & 0.865 \\
\hline K-ABC Sequential Processing ${ }^{a}$ & $104.13^{\mathrm{a}}(15.71)$ & $94.25^{\mathrm{a}}(10.65)$ & -0.70 & 0.127 \\
\hline K-ABC Simultaneous Processing & $108.86^{\mathrm{b}}(14.36)$ & $101.50(9.06)$ & -0.57 & 0.208 \\
\hline K-ABC Mental Processing Composite & $108.21^{\mathrm{b}, \mathrm{c}}(14.49)$ & $98.25^{\mathrm{c}}(8.86)$ & -0.77 & 0.094 \\
\hline WRAML Verbal Memory & $103.87(14.72)$ & $96.88(17.07)$ & -0.45 & 0.316 \\
\hline WRAML Visual Memory & $91.53(17.15)$ & $92.00(14.77)$ & 0.03 & 0.949 \\
\hline WRAML Learning & $103.07(14.87)$ & $96.13(18.73)$ & -0.43 & 0.341 \\
\hline WRAML General Memory & $99.47(17.14)$ & $93.75(17.72)$ & -0.33 & 0.460 \\
\hline \multicolumn{5}{|l|}{ Girls without $\mathrm{CAH}$} \\
\hline$n$ & 31 & 35 & & \\
\hline Age at test (years) & $8.38(2.42)$ & $8.04(2.29)$ & -0.14 & 0.560 \\
\hline Midparental education & $5.62^{\mathrm{d}}(1.08)$ & $5.49(1.00)$ & -0.13 & 0.614 \\
\hline K-ABC Sequential Processing ${ }^{a}$ & $104.77^{\mathrm{a}}(13.73)$ & $108.03^{a}(10.49)$ & 0.26 & 0.280 \\
\hline K-ABC Mental Processing Composite & $108.87^{\mathrm{C}}(12.21)$ & $111.06^{\mathrm{c}}(9.72)$ & 0.20 & 0.421 \\
\hline K-ABC Achievement & $107.35(10.01)$ & $105.51(12.03)$ & -0.17 & 0.505 \\
\hline WRAML Verbal Memory & $101.30^{\mathrm{d}}(12.16)$ & $101.49(13.34)$ & 0.02 & 0.954 \\
\hline WRAML Visual Memory & $97.10(13.32)$ & $96.51(14.06)$ & -0.04 & 0.864 \\
\hline WRAML Learning & $105.32(12.54)$ & $102.49(14.91)$ & -0.20 & 0.409 \\
\hline WRAML General Memory & $101.37^{d}(12.41)$ & $100.23(14.92)$ & -0.09 & 0.742 \\
\hline \multicolumn{5}{|l|}{ Boys with $\mathrm{CAH}$} \\
\hline$n$ & 11 & 8 & & \\
\hline Age at test (years) & $8.28(1.88)$ & $7.60(1.63)$ & -0.38 & 0.421 \\
\hline Midparental education & $5.41(1.28)$ & $5.50(0.85)$ & 0.08 & 0.864 \\
\hline K-ABC Sequential Processing ${ }^{\mathrm{e}}$ & $97.82^{\mathrm{e}}(8.72)$ & $101.25^{\mathrm{e}}(7.57)$ & 0.41 & 0.384 \\
\hline K-ABC Simultaneous Processing & $106.09(14.17)$ & $111.88(12.12)$ & 0.43 & 0.365 \\
\hline K-ABC Mental Processing Composite ${ }^{f}$ & $103.00^{f}(12.92)$ & $108.13^{f}(11.22)$ & 0.42 & 0.380 \\
\hline K-ABC Achievement ${ }^{\mathrm{g}}$ & $101.91^{\mathrm{g}}(11.50)$ & $107.63^{\mathrm{g}}(13.03)$ & 0.47 & 0.326 \\
\hline WRAML Verbal Memory & $93.73^{\mathrm{h}}(6.78)$ & $95.86(9.03)$ & 0.28 & 0.575 \\
\hline WRAML Visual Memory & $94.55(13.19)$ & $100.63(9.21)$ & 0.51 & 0.280 \\
\hline WRAML Learning & $95.45(11.20)$ & $103.00(13.29)$ & 0.63 & 0.197 \\
\hline WRAML General Memory & $93.18^{\mathrm{h}}(9.26)$ & $99.57(10.36)$ & 0.66 & 0.191 \\
\hline \multicolumn{5}{|l|}{ Boys without $\mathrm{CAH}$} \\
\hline Midparental education & $5.84(1.06)$ & $5.47(0.96)$ & -0.37 & 0.302 \\
\hline K-ABC Sequential Processing ${ }^{e}$ & $99.44^{\mathrm{e}}(11.82)$ & $106.19^{\mathrm{e}}(10.24)$ & 0.62 & 0.095 \\
\hline K-ABC Simultaneous Processing & $109.50(11.04)$ & $113.25(10.02)$ & 0.35 & 0.323 \\
\hline K-ABC Mental Processing Composite ${ }^{f}$ & $106.06^{f}(11.02)$ & $111.75^{f}(9.68)$ & 0.55 & 0.132 \\
\hline K-ABC Achievement ${ }^{\mathrm{g}}$ & $104.25^{\mathrm{g}}(16.82)$ & $112.88^{\mathrm{g}}(13.60)$ & 0.57 & 0.121 \\
\hline WRAML Verbal Memory & $97.88(16.77)$ & $100.63(14.66)$ & 0.17 & 0.625 \\
\hline WRAML Visual Memory & $92.88(13.81)$ & $97.50(13.59)$ & 0.34 & 0.347 \\
\hline WRAML Learning & $102.88(14.32)$ & $106.25(11.86)$ & 0.25 & 0.473 \\
\hline WRAML General Memory & $97.56(15.66)$ & $101.75(11.53)$ & 0.31 & 0.396 \\
\hline \multicolumn{5}{|l|}{ Non-CAH girls and boys combined } \\
\hline$n$ & 47 & 51 & & \\
\hline Age at test (years) & $8.25(2.29)$ & $8.17(2.26)$ & -0.04 & 0.879 \\
\hline Midparental education & $5.70^{i}(1.07)$ & $5.48(0.98)$ & -0.21 & 0.303 \\
\hline K-ABC Sequential Processing & $102.96(13.23)$ & $107.45(10.35)$ & 0.38 & 0.066 \\
\hline K-ABC Simultaneous Processing & $109.91(12.16)$ & $111.67(9.22)$ & 0.17 & 0.427 \\
\hline K-ABC Mental Processing Composite & $107.91(11.77)$ & $111.27(9.62)$ & 0.32 & 0.124 \\
\hline K-ABC Achievement & $106.30(12.64)$ & $107.82(12.88)$ & 0.12 & 0.556 \\
\hline WRAML Verbal Memory & $100.11^{i}(13.85)$ & $101.22(13.63)$ & 0.08 & 0.693 \\
\hline WRAML Visual Memory & $95.66(13.49)$ & $96.82(13.79)$ & 0.08 & 0.674 \\
\hline WRAML Learning & $104.49(13.07)$ & $103.67(14.02)$ & -0.06 & 0.765 \\
\hline WRAML General Memory & $100.04^{i}(13.58)$ & $100.71(13.84)$ & 0.05 & 0.813 \\
\hline
\end{tabular}

$P$ values in bold indicate marginal $(P<0.10)$ or conventional $(P<0.05)$ significance.

Sample size reductions due to missing data

${ }^{\mathrm{b}} n=14$.

${ }^{d} n=30$.

${ }^{\mathrm{h}} n=7$.

${ }^{\mathrm{i}} n=46$

Results of hierarchical multiple regression analysis where marginally or conventionally significant:

${ }^{a} \mathrm{CAH}$ effect $(P=0.071)$, DEX effect not significant (NS), CAH $\times$ DEX interaction $(P=0.042)$.

${ }^{\mathrm{c}} \mathrm{CAH}$ effect $(P=0.059)$, DEX effect NS, CAH $\times \mathrm{DEX}$ interaction $(P=0.039)$.

${ }^{\circ} \mathrm{CAH}$ effect NS, DEX effect $(P=0.057), \mathrm{CAH} \times \mathrm{DEX}$ interaction NS.

${ }^{\dagger} \mathrm{CAH}$ effect NS, DEX effect $(P=0.082), \mathrm{CAH} \times \mathrm{DEX}$ interaction NS.

${ }^{\mathrm{g}} \mathrm{CAH}$ effect NS, DEX effect $(P=0.063), \mathrm{CAH} \times \mathrm{DEX}$ interaction NS. 
Re-analysis of the data with adjustment for K-ABC Achievement as IQ estimate yielded slight increases in $P$ values and, thereby, reduction of the number of (marginally or conventionally) significant variables by nine and, conversely, slight decreases in $P$ values and, thereby, the addition of five newly (marginally or conventionally) significant variables, but did not affect the overall conclusions (not shown). For results of Study 2, see Supplementary Study and Table 2 (see section on supplementary data given at the end of this article).

\section{Discussion}

The vast majority of group comparisons in Study 1 (Table 1 and Supplementary Table 1, see section on supplementary data given at the end of this article) were not significant, neither conventionally nor marginally. Thus, our data do not indicate a strong broadband effect of early-onset prenatal DEX exposure on mental processing and memory performance in childhood. However, the pattern of few significant findings suggests a difference in effect between short- and long-term exposures during pregnancy. Except for one conventionally significant result, most findings for short-term exposure (five marginally and three conventionally significant) show better performance for the DEX exposed compared with controls, while the long-term findings (three marginally significant) show worse performance for the DEX exposed. This contrast is further strengthened by the results of the hierarchical regression analyses and even maintained - albeit weakened - when adjusted for IQ. The small data set from Study 2 shows a similar pattern.

Thus, if there is indeed a replicable adverse effect of early-onset prenatal DEX exposure, it appears to be more likely with long-term exposure. Our data suggest that the $\mathrm{K}-\mathrm{ABC}$ measures of both sequential and simultaneous processing may be particularly sensitive to longterm DEX exposure. On the other hand, the positive partial correlations of cognitive data with DEX exposure duration in Study 1 (two in the total sample, namely, WRAML Visual Memory and K-ABC Faces \& Places, and three in the non-CAH sample, namely, WRAML Visual Memory, WRAML General Memory Index, and K-ABC Faces \& Places; of those five, three marginally and two conventionally significant) do not support an adverse effect of DEX exposure on the neuropsychological variables assessed here. The one negative correlation, with K-ABC Reading/Understanding, in the subsample with $\mathrm{CAH}$ includes only five patients and is therefore hardly interpretable. This finding, along with the low statistical power of our long-term exposure comparisons, indicates that, while raising concerns, our findings on long-term prenatal DEX exposure are not definitive.

Despite selecting a battery of tests emphasizing mental processing and memory performance, we did not corroborate the findings by Hirvikoski et al. (12) of adverse effects of short-term prenatal DEX exposure on memory. Several explanations appear plausible. i) Short-term prenatal DEX affects only the specific working memory ability covered by the Wechsler Digit Span test, i.e. both forward and backward digit recall, not just the working memory's short-term memory component involved in forward digit span. This would be a very narrow impairment indeed, but this possibility cannot be currently excluded. On the other hand, one needs to keep in mind that Wechsler Digit Span is an incomplete measure of working memory as currently conceptualized $(23,24)$; only a battery of experimental procedures can comprehensively assess all its individual components. ii) In the US health care system, DEX treatment of CAH-risk pregnancies implies a positive socioeconomic bias associated with cognitive ability or educational level favoring the DEX exposed over controls. However, such a selection bias is not indicated by the data on midparental education and IQ in Study 1 (but may play a role in the very small Study 2). iii) Among males with $\mathrm{CAH}$, prenatal diagnosis and prenatal DEX treatment usually imply immediate postnatal initiation of glucocorticoid and mineralocorticoid treatment, as appropriate, while in the control males with $\mathrm{CAH}$, the diagnosis at birth and start of treatment are more likely delayed with the attendant risks of electrolyte crises and their potentially adverse effects on the developing brain $(2,25,26,27)$ (of course, even in prenatally diagnosed and treated children with $\mathrm{CAH}$, postnatal electrolyte crises may occur due to lapses in treatment adherence or failure to increase the medication dose during illness). In any case, this rationale does not apply to the non-CAH groups. iv) The least plausible explanation is that shortterm prenatal DEX exposure actually improves cognitive outcome. We are not aware of an evidence-based rationale that would support such a hypothesis.

Our follow-up studies have two significant limitations, as is typical of all studies to date in this area. One is that DEX treatment was not prescribed in the format of a randomized clinical trial. Instead, we conducted an observational follow-up study of a clinical treatment sample and, in Study 1, could rule out a decisive influence of the most obvious potentially confounding variables: age, midparental education, and IQ. The other significant limitation is that, because of financial constraints, only a part of the total DEXexposed sample could be included. This fact limited the sample size and thereby the statistical power of the group comparisons. It is not clear, however, whether it led to any biases in our findings.

In conclusion, our studies do not replicate an earlier report of adverse effects of short-term prenatal DEX exposure on working memory, while our finding of lower cognitive processing in $\mathrm{CAH}$ girls and women with long-term DEX exposure contributes to concerns about potentially adverse cognitive after effects of such 
exposure. Yet, our studies are not definitive, and replications in larger samples are required.

\section{Supplementary data}

This is linked to the online version of the paper at http://dx.doi.org/10. 1530/EJE-11-0789.

\section{Declaration of interest}

The authors declare that there is no conflict of interest that could be perceived as prejudicing the impartiality of the research reported.

\section{Funding}

This work was supported in part by NICHD grant R01 HD00072-33A (M I New), NCRR grant U54 RR019484 (M I New), and NIMH grant P30 MH43520 (Anke A Ehrhardt). The content is solely the responsibility of the authors and does not necessarily represent the official views of the NICHD, NCRR, NIMH, or the NIH.

\section{Acknowledgements}

In Study 1, Susan W Baker, PhD, served as study coordinator and test performance monitor; Jihad S Obeid, MD, and Ann D Carlson, MA, worked on data bank maintenance and sample selection; Leah Enfield, BA, and Bonnie Sihler, BA, administered the neuropsychological tests: Lauren Beamud performed the financial administration; and Nadina Canellopoulou, BA, Joseph Anastasio, Caitlin Rea, Gabriella Neuwirth, and Vanessa Cunanan served as research assistants. In Study 2, Susan W Baker served as study coordinator and worked, along with Jihad $S$ Obeid, on sample selection; Sarah Dumais performed the financial administration; and Jessica Kaltman, BA, Trishna Nath, BA, Cliff Hamilton, BA, and Zachary Lerner, BA, served as research assistants. Cheng-Shiun Leu, PhD, provided statistical consultation. The authors also thank three anonymous reviewers for their contribution.

\section{References}

1 Frisén L, Nordenström A, Falhammar H, Filipsson H, Holmdahl G, Janson PO, Thorén M, Hagenfeldt K, Möller A \& Nordenskjöld A. Gender role behavior, sexuality, and psychosocial adaptation in women with congenital adrenal hyperplasia due to CYP21A2 deficiency. Journal of Clinical Endocrinology and Metabolism 200994 3432-3439. (doi:10.1210/jc.2009-0636)

2 Meyer-Bahlburg HFL. Brain development and cognitive, psychosocial, and psychiatric functioning in classical 21-hydroxylase deficiency (21-OHD). Endocrine Development 201120 88-95. (doi:10.1159/000321225)

3 David M \& Forest MG. Prenatal treatment of congenital adrenal hyperplasia resulting from 21-hydroxylase deficiency. Journal of Pediatrics $1984 \quad 105 \quad 799-803 . \quad$ (doi:10.1016/S00223476(84)80310-8)

4 New MI, Carlson A, Obeid J, Marshall I, Cabrera MS, Goseco A, Lin-Su K, Putnam AS, Wei JQ \& Wilson RC. Prenatal diagnosis for congenital adrenal hyperplasia in 532 pregnancies. Journal of Clinical Endocrinology and Metabolism 200186 5651-5657. (doi:10.1210/jc.86.12.5651)

5 Speiser PW, Azziz R, Baskin LS, Ghizzoni L, Hensle TW, Merke DP, Meyer-Bahlburg HFL, Miller WL, Montori VM, Oberfield SE, Ritzen M \& White PC. Congenital adrenal hyperplasia due to steroid 21-hydroxylase deficiency: an Endocrine Society Clinical Practice Guideline. Journal of Clinical Endocrinology and Metabolism 201095 4133-4160. (doi:10.1210/jc.2009-2631)

6 Fernández-Balsells MM, Muthusamy K, Smushkin G, Lampropulos JF, Elamin MB, Abu Elnour NO, Elamin KB,
Agrwal N, Gallegos-Orozco JF, Lane MA, Erwin PJ, Montori VM \& Murad MH. Prenatal dexamethasone use for the prevention of virilization in pregnancies at risk for classical congenital adrenal hyperplasia because of 21-hydroxylase (CYP21A2) deficiency: a systematic review and meta-analysis. Clinical Endocrinology 2010 73 436-444. (doi:10.1111/j.1365-2265.2010.03826.x)

7 Hauser J, Knapman A, Zürcher NR, Pilloud S, Maier C, Diaz-Heijtz R, Forssberg H, Dettling A, Feldon J \& Pryce CR. Effects of prenatal dexamethasone treatment on physical growth, pituitary-adrenal hormones, and performance of motor, motivational, and cognitive tasks in juvenile and adolescent common marmoset monkeys. Endocrinology $2008 \quad 149$ 6343-6355. (doi:10.1210/en.2008-0615)

8 Seckl JR. Glucocorticoids, developmental 'programming' and the risk of affective dysfunction. Progress in Brain Research 2008167 17-34. (doi:10.1016/S0079-6123(07)67002-2)

9 Tegethoff M, Pryce Ch \& Meinlschmidt G. Effects of intrauterine exposure to synthetic glucocorticoids on fetal, newborn, and infant hypothalamic-pituitary-adrenal axis function in humans: a systematic review. Endocrine Reviews 200930 1-37. (doi:10.1210/er.2008-0014)

10 Trautman PD, Meyer-Bahlburg HFL, Postelnek J \& New MI. Effects of early prenatal dexamethasone on the cognitive and behavioural development of young children: results of a pilot study. Psychoneuroendocrinology $1995 \quad 20$ 439-449. (doi:10.1016/ 0306-4530(94)00070-0)

11 Meyer-Bahlburg HF, Dolezal C, Baker SW, Carlson AD, Obeid JS \& New MI. Cognitive and motor development of children with and without congenital adrenal hyperplasia after early-prenatal dexamethasone. Journal of Clinical Endocrinology and Metabolism 200489 610-614. (doi:10.1210/jc.2002-021129)

12 Hirvikoski T, Nordenström A, Lindholm T, Lindblad F, Ritzén EM, Wedell A \& Lajic S. Cognitive functions in children at risk for congenital adrenal hyperplasia treated prenatally with dexamethasone. Journal of Clinical Endocrinology and Metabolism 2007 92 542-548. (doi:10.1210/jc.2006-1340)

13 Hirvikoski T, Nordenström A, Lindholm T, Lindblad F, Ritzén EM \& Lajic S. Long-term follow-up of prenatally treated children at risk for congenital adrenal hyperplasia: does dexamethasone cause behavioural problems? European Journal of Endocrinology 2008 159 309-316. (doi:10.1530/EJE-08-0280)

14 Meyer-Bahlburg H, Dolezal C, Baker S, Carlson A, Obeid J \& New M. Prenatal androgenization affects gender-related behavior but not gender identity in 5-12 year-old girls with congenital adrenal hyperplasia. Archives of Sexual Behavior 200433 97-104. (doi:10.1023/B:ASEB.0000014324.25718.51)

15 Hollingshead A. Four Factor Index of Social Status (unpublished manuscript). New Haven, CT: Yale University, Department of Sociology, 1975.

16 Kaufman AS \& Kaufman NL. Kaufman Assessment Battery for Children. Circle Pines, MN: American Guidance Service, 1983.

17 Kline RB, Snyder J \& Castellanos M. Lessons from the Kaufman Assessment Battery for Children (K-ABC): toward a new cognitive assessment model. Psychological Assessment $1996 \quad 4 \quad 7-17$. (doi:10.1037/1040-3590.8.1.7)

18 Sheslow D \& Adams W. Wide Range Assessment of Memory and Learning. Wilmington, DE: Wide Range, Inc., 1990.

19 Psychological corporation. Wechsler Abbreviated Scale of Intelligence (WASI). San Antonio, TX: Author, 1999.

20 Sheslow D \& Adams W. Wide Range Assessment of Memory and Learning, 2nd edn. Lutz, FL: Psychological Assessment Resources, Inc., 2003.

21 Wechsler D. Wechsler Adult Intelligence Scale, 3rd edn, San Antonio, TX: Psychological Corporation, 1997.

22 Wechsler D. Wechsler Intelligence Scale for Children, 4th edn, San Antonio, TX: Psychological Corporation, 2003.

23 Cowan N. Multiple concurrent thoughts: the meaning and developmental neuropsychology of working memory. Developmental Neuropsychology $2010 \quad 35$ 447-474. (doi:10.1080/ 87565641.2010.494985) 
24 Khan ZU \& Muly EC. Molecular mechanisms of working memory. Behavioral Brain Research 2011219 329-341. (doi:10.1016/j. bbr.2010.12.039)

25 Donaldson MDC, Thomas PH, Love JG, Murray GD, McNinch AW \& Savage DCL. Presentation, acute illness, and learning difficulties in salt wasting 21-hydroxylase deficiency. Archives of Disease in Childhood 199470 214-218. (doi:10.1136/adc.70.3.214)

26 Johannsen TH, Ripa CPL, Reinisch JM, Schwartz M, Mortensen EL \& Main KM. Impaired cognitive function in women with congenital adrenal hyperplasia. Journal of Clinical Endocrinology and Metabolism 200691 1376-1381. (doi:10.1210/jc.2005-1959)
27 Somajni F, Sovera V, Albizzati A, Russo G, Peroni P, Seragni G \& Lenti C. Neuropsychological assessment in prepubertal patients with congenital adrenal hyperplasia: preliminary study. Minerva Pediatrica $2011631-9$.

Received 7 September 2011

Revised version received 16 April 2012

Accepted 1 May 2012 